\title{
e-Phaïstos
}

e-Phaïstos

Revue d'histoire des techniques / Journal of the history

of technology

IX-1 | 2021

Autour de Léonard de Vinci

\section{«Une fureur sacrée de comprendre pour faire et de faire pour comprendre »}

Le témoignage de Léonard de Vinci

"A sacred Fury to Understand in Order to Do and to Do in Order to Understand". The Testimony of Leonardo da Vinci

Jean-Louis Le Moigne

\section{OpenEdition}

Journals

Édition électronique

URL : https://journals.openedition.org/ephaistos/8975

DOI : 10.4000/ephaistos.8975

ISSN : 2552-0741

Éditeur

IHMC - Institut d'histoire moderne et contemporaine (UMR 8066)

Référence électronique

Jean-Louis Le Moigne, « « Une fureur sacrée de comprendre pour faire et de faire pour comprendre » », e-Phaïstos [En ligne], IX-1 | 2021, mis en ligne le 27 avril 2021, consulté le 25 septembre 2021. URL http://journals.openedition.org/ephaistos/8975 ; DOI : https://doi.org/10.4000/ephaistos.8975

Ce document a été généré automatiquement le 25 septembre 2021.

Tous droits réservés 


\section{«Une fureur sacrée de comprendre pour faire et de faire pour comprendre »}

Le témoignage de Léonard de Vinci

"A sacred Fury to Understand in Order to Do and to Do in Order to Understand". The Testimony of Leonardo da Vinci

Jean-Louis Le Moigne

1 C'est à dessein que j'introduis par ce titre insolite emprunté à Paul Valéry (présentant en 1939 la parution de la traduction en français des Carnets de Léonard qu'il préfaçait par ailleurs) quelques arguments pouvant retenir l'attention des historiens des techniques et celle des enseignants-chercheurs en "Technologie », disciplines parfois qualifiées de 'sciences appliquées', libellé valorisant souvent le statut académique des enseignants en «technologie» - 'science ancillaire', dit-on parfois - mais le dévalorisant en ceci qu'on les invite à «faire appliquer pratiquement» des connaissances de forme théorique déjà formulées plutôt qu'à s'exercer à concevoir et comprendre ces connaissances théoriques (qualifiées souvent de 'sciences fondamentales'). Ceci alors que ces enseignants souhaitent aider leurs élèves et étudiants à « Comprendre en s'exerçant cognitivement à Concevoir ».

2 Placé dans cette Revue d'Histoire des techniques se formant sous le signe du « Disque de Phaïstos ", découvert en 1908, encore tenu pour unique, daté approximativement alentour de $1800 \mathrm{BC}$, et des 45 poinçons permettant de générer 241 symboles sur les deux faces de ce Disque, 'système de symboles' que l'on cherche encore à interpréter et comprendre, le titre -et le contexte- de cet article sera sans doute moins insolite?

3 N'est-ce pas cette 'passion de comprendre' dont nous percevons la prégnance dans nos cultures contemporaines de l'ouvrage -et- de l'œuvre écrite considérable de Léonard de Vinci qui, depuis plus de 150 ans, anime experts et historiens (je n'ose dire archéologues) retrouvant, traduisant et éditant peu à peu les 7000 pages des Carnets et autres écrits retrouvés de Léonard actuellement aisément accessibles (Le Codex de 
Madrid, le plus récemment retrouvé en 1965, est ainsi ignoré de la réédition en français des Carnets de Léonard en format poche, Gallimard ayant repris l'édition originale de 1942).

Pour ne pas manquer les pages de ce Codex consacrées principalement à des 'Technologies' (sous ce label dont Léonard récuserait la légitimité disciplinaire, on trouve nombre d'études associant sciences, récits et dessins d'expériences relevant de la mécanique, de l'horlogerie, de l'architecture militaire et engins de guerre, ou de l'hydraulique des égouts et canaux, par exemple), on pourra faire appel au volume consacré par Ladislao Reti en 1974 (éd. R Laffont), à Léonard de Vinci, l'Artiste, l'Inventeur, Humaniste, (alors que le titre original en anglais est "The Unknown Leonardo » : s'il est légitime de convenir de la pertinence du qualificatif 'Inconnu' pour l'acheteur francophone, il ne l'est pas de réduire la traduction du mot à trois catégories socioprofessionnelles).

5 Cette rapide évocation de l'œuvre et de l'ouvrage risque pourtant de nous mettre en situation de privilégier la lecture du point de vue du seul 'maître d'ouvrage' plutôt qu'à celle du 'maître d'œuvre' -le premier s'intéressant à la qualité des 'résultats' (attitude plausible et habituellement retenue par les critiques artistiques ou scientifiques). On comprend alors pourquoi les académies scientifiques classent volontiers l'ouvrage écrit de Léonard dans la catégorie des 'Technologies', tenue pour souvent synonyme des 'sciences appliquées' et tardent à accepter l'expression 'Sciences de l'Artificiel', argumentée par H. A. Simon depuis 1963.

6 C'est pourtant l'autre point de vue, celui du 'maître d'œuvre', que les pages des Carnets mêlant dessins, schémas et textes descriptifs ou narratifs, souvent associant de mémoire d'autres références, que Léonard va surtout privilégier, certes sans exclusive. Son possible autoportrait (fig.1) méditant sur la formation des tourbillons dans un ruisseau pour interpréter les phénomènes hydrauliques prend ici une valeur symbolique : celle du "chercheur praticien réfléchissant», tentant d'inférer des lois plausibles de la dynamique de l'hydraulique, sur le terrain en notant sur-le-champ ses observations-descriptions-conceptions de nouvelles expérimentations à envisager: « L'Expérience est la mère de la connaissance (Sapiença) » écrira-t-il sur un Carnet. 
Fig.1. Vieil homme de profil, assis sur le rebord d'un rocher ; études d'eau, Léonard de Vinci

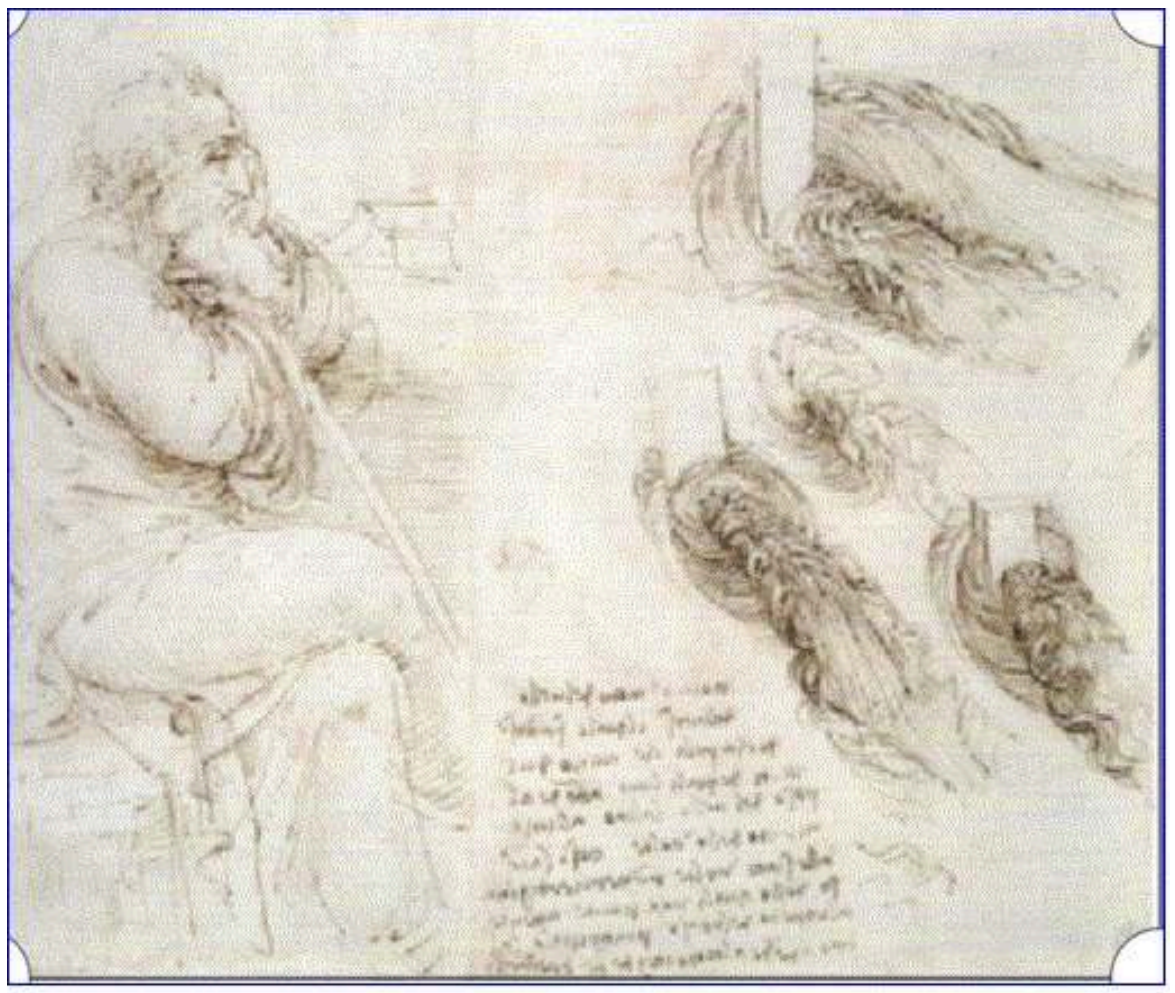

Plume et encre, 152 × 213 mm, vers 1510-1513, Royal Library (RL 12579r), Windsor castle

7 Attitude de maître d'œuvre concepteur plutôt que d'analyste, et intentionnellement projectif plutôt que se présumant froidement objectif, préférant le 'Fonctionnel' au 'Formel' dans ses descriptions d'expérimentation; il faudra pourtant attendre les années 1960 pour que quelques autorités académiques telles que J. Piaget, H. Simon ou E. Morin le rappellent: "On en vient de plus en plus aujourd'hui à considérer la connaissance comme un processus que comme un état» (Piaget 1970:79). Mais la culture du «Focus on Process» (« La méditation de l'objet par le Sujet prend toujours la forme du Projet », Bachelard 1934) infuse encore très lentement dans les programmes d'enseignement, souvent inattentifs aux ressources de la Problématisation par Rhétorique et Topique et plus généralement par les sciences de l'Argumentation. On sait comment faire une copie du Disque de Phaïstos, mais on ne comprend pas (encore) pourquoi et dans quel contexte 'un sujet formant consciemment projet' l'a fait.

$8 \mathrm{Ne}$ peut-on pourtant tenter de comprendre en s'attachant à associer intentionnellement la forme et la fonction, dans la conception et dans l'interprétation des connaissances humaines? On retrouve ici sans surprise les termes de la doctrine dite fonctionnaliste de l'architecture, «les Trois $F$ : Form follows function ", présentation fréquemment privilégiée et généralisée dans nombre de processus de Conception -le 'Designing'.

9 La prise de conscience et l'identification de la dialogique de 'la Forme' attribuée à l'Objet perçu ou conçu et des 'Fonctions endo- et exogènes' identifiables devant ou pouvant être assurée par le 'Sujet', va permettre la formation et la transformation des connaissances humaines par les jeux d'activités cognitives en interaction de sujets s'attachant d'abord à comprendre comme de ceux s'attachant d'abord à faire. Il s'agira 
toujours de veiller à associer intentionnellement la représentation dite d'état et la représentation dite de processus $^{1}$ dans la formation et la transformation des connaissances humaines. H. A. Simon note, à partir de 1962 : «L'idée consistant à substituer une description de processus à une description d'état a joué un rôle central dans le développement de la science moderne ». Ce qui le conduisit à mettre en valeur "l'unité des arts et des sciences, la psychologie de la pensée et la découverte ${ }^{2}$ » dans un article présenté à l'American Academy of Arts and Sciences en mai 1981.

10 L'exercice est plausible. On peut ici s'arrêter un instant pour prêter attention à l'article rédigé en 1894 par un jeune homme de 23 ans, Paul Valéry, qui avait pu accéder aisément aux premières études et traductions françaises des Carnets de Léonard (Séailles 1892, Ravaisson-Mollien 1894), article édité par La Nouvelle revue, sous un titre a priori audacieux : «Introduction à la Méthode de Léonard de Vinci ${ }^{3}$ ».

11 Un siècle plus tard, ce texte deviendra un des trois textes les plus fameux de Paul Valéry. Celui-ci témoignera de la légitimité de son audace en se référant implicitement, dans ses Cahiers et dans la plupart de ses nombreux essais publiés jusqu'à sa mort (1945), à des arguments et commentaires qui traduisent souvent une attention voisine à celle des arguments relevés dans les pages des Carnets de Léonard, édités en 1973 et en 2016. La lecture des cahiers sur lesquels Valéry rédigeait chaque matin depuis 1894, ses méditations du moment dans des contextes évoluant, en s'attachant à 'comment son esprit fonctionne' lorsqu'il s'essaye à 'faire attention' pour tenter intentionnellement de comprendre, tout en convenant : «J'ai beau faire, tout m'intéresse ».

Les exégètes contemporains de Léonard comme ceux de $\mathrm{P}$. Valéry en conviendront: n'est-ce pas la même interpellation qu'ils entendent lorsqu'ils tentent d'organiser par disciplines contemporaines les notes s'accumulant sur les Carnets de Léonard ? Ceux-ci leur répondent volontiers: "Pour comprendre cet arbre, tu ne peux le séparer de quelque sorte de fond sur lequel il se détache ».

13 Mais si les 'faire' diffèrent dans des contextes si différents (des années 1490 aux années 1890), ils demeurent effectivement des processus observation-description-conception construits dans les deux cas sur des projets intentionnels, dont on enregistre ici la trace sur une page de Carnet ou de Cahier. Trace qui pourra s'avérer problématisante, parfois catalysante pour éclairer la Théorie de la connaissance humaine qui continue à se transformer 'à l'expérience'. J'illustre ceci en empruntant à chacun une citation qui mérite, me semble-t-il, un regain d'attention dans nos cultures.

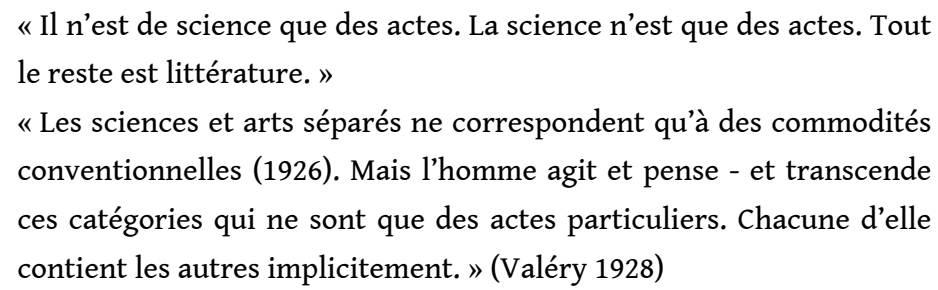

Je ne sais si H. A. Simon connaissait ce texte de Valéry lorsqu'il présenta en 1959 à l'Académie des sciences et des arts des États-Unis, dont il était membre, sa contribution intitulée «L'unité des arts et des sciences: la psychologie de la pensée et de la découverte ».

15 Ne doit-on pas aujourd'hui convenir que les réflexions de Léonard de Vinci, pour qui aucun des nombreux 'champs d'attention' (je voudrai ici éviter le mot 'discipline' que 
ne connaissait pas Léonard) au sein desquels il avait développé modélisations, expérimentations et conceptions plausibles, ne relèvent pas d'activités cognitives à fins méthodologiques notablement différentes de celles que l'usage attribue aux Sciences et aux Arts ? Si l'on peut parler de « la science de la peinture » dans les termes retenus par André Chastel (2012), ne pourra-t-on tenir l'invention de la connaissance du sfumato par Léonard pour connaissance scientifique au moins autant que pour artistique, connaissance épistémologiquement désormais bien argumentée ?

La démarche de «L'Introduction à la Méthode de Léonard de Vinci » mise en valeur par la problématisation originale proposée par P. Valéry, passant de l'usuel 'Objectif' : «Comment la Nature fonctionne » au 'Projectif' : «Comment mon Esprit fonctionne » rejoint ici celle que H. A. Simon rappellera en tenant pour connaissance légitime une connaissance humaine reliante formée sur ce référentiel épistémologico-critique.

En publiant en 1987 une étude judicieusement intitulée Les inventions de la Nature et la nature de l'Invention, Martin Kemp nous invite à être attentif à la conscience de ces deux faces de notre connaissance de l'invention formant ou transformant les connaissances. Thèse qu'il argumente en mentionnant trois brefs extraits des Carnets que l'on reprend ici :

\begin{abstract}
«Au point où la nature s'arrête de produire ses espèces, l'homme, avec les choses naturelles, crée à l'aide de cette nature une variété infinie d'espèces » (RL 19030v; K/P72v).

"L'homme est le principal instrument de la nature, car elle ne s'occupe que de la production des corps élémentaires dont l'homme extrait un nombre illimité de composés, bien qu'il n'ait pas le pouvoir de créer des corps naturels hormis d'autres semblables à lui-même, c'est-à-dire ses enfants » (RL $19045 \mathrm{v} ; \mathrm{K} / \mathrm{P}$ 50).

«La représentation [Disegno] est d'une excellence telle qu'elle ne fait pas que montrer les œuvres de la nature, mais qu'elle en produit un nombre infiniment plus varié... et, à cause de cela, nous concluons que ce n'est pas seulement une science, mais un pouvoir divin méritant ses lettres de noblesse. ( $\mathrm{CU}, £ 50 \mathrm{r}$ ). Elle surpasse la nature parce que les formes élémentaires de la nature sont limitées tandis que les œuvres que l'œil exige des mains de l'homme sont illimitées » (CU, f. 116 r) » (Kemp 1987).
\end{abstract}

L'invention de la connaissance est alors perçue par un 'travail de l'esprit', auquel P. Valéry va souvent s'attacher, contribuant implicitement à la formation potentielle dans le champ de la connaissance par la progressive émergence de la science de la cognition, autrement dit par l'étude des processus cognitifs de formation-transformation de connaissance. Ceux-ci s'exprimant indirectement dans les Carnets de Léonard inciteront le jeune Paul Valéry à écrire son insolite "Introduction à la Méthode de Léonard de Vinci " puis, rencontrant d'abord 'le problème du langage', à adapter sous des formes et dans des contextes différents, le procédé des notes rédigées au vol sur un carnet ou sur un cahier. Si bien que nous disposons de l'édition des Cahiers colligée et dirigée par Judith Robinson qui fut la directrice de la première édition des Cahiers de Valéry (NRF-Pléiade 1973), une énorme masse de notes remplissant 261 Cahiers de formats divers. 
19 En se livrant à cet exercice d'édition du volumineux manuscrit des Cahiers de Valéry, J. Robinson eut l'heureuse idée de s'exercer à une sorte d'Introduction à la Méthode de Paul Valéry telle qu'on peut la percevoir en particulier dans les Cahiers de Valéry, ceci dans une intention voisine de celle qui motivait Paul Valéry 80 ans plus tôt. Ce sera L'analyse de l'esprit dans les Cahiers de Valéry (Robinson 1973).

\section{« La représentation [Disegno] est d'une excellence telle que... »}

Je me proposais, en entreprenant cet article, de revenir sur 'le problème de langage' que pose le thème de ce numéro IX-1 présentant Léonard de Vinci en tant que Grand Penseur de la Technique. Je m'interrogeai sur la réduction de la pensée de Léonard et celle des connaissances qu'elle forme et transforme la seule technique et j'envisageai, à partir de cette problématisation, de reconsidérer les représentations que nous formons actuellement de la technique sous-jacente à cette qualification pour Léonard ? Ce qui aurait dû me conduire à examiner les représentations gnoséologique et épistémologique d'autres penseurs. Ceci en notant que Léonard et ses contemporains ne disposaient pas des mots 'Technique' et 'Technologique'. Mais ils disposaient du concept très riche de 'Disegno' qui, en italien, exprime la dialogique du dessein et du dessin. Le 'Disegno' engage le praticien-réfléchissant comme le chercheurexpérimentant dans la Trans-Formation de connaissances activantes.

Réflexion qui conduira à 'La Question' à laquelle se consacra Paul Valéry dans ses Cahiers : «Comment mon Esprit fonctionne?»; question qu'il argumentera de plus en plus par un diagnostic qu'il soulignera encore en 1948, dans Vues, et qui mérite d'être rappelée : "Qu'est-il arrivé ? Il est arrivé que nos moyens d'investigation et d'action laissent loin derrière eux nos moyens de représentations et de compréhension ». Nous devenant familier, cet argument nous incitera sans doute à associer aux recherches dites technologiques, les recherches dans le champ des sciences de la cognition qui appellent de nouvelles formes 'd'épistémologie critique' dans les explorations des 'Champs des Possibles'. N'est-ce pas ainsi qu'émergent aujourd'hui les sciences d'Ingenium, sciences du Génie, sciences de Conception, sciences de l'Artificiel ?

Je ne surprendrai pas les historiens de la technique ici en m'efforçant de tenir avec eux le viatique de Blaise Pascal: "Travailler à bien penser, voilà la source de l'éthique ». Nous dit-il autre chose que Léonard rappelant volontiers sa célèbre devise "Ostinato Rigore»?

\section{BIBLIOGRAPHIE}

BACHELARD Gaston, Le nouvel esprit scientifique, Paris, PUF, 1934

CHASTEL André, Léonard ou les sciences de la Peinture, Paris, Liana Levi, 2012 
KEMP Martin, Les inventions de la Nature et la nature de l'Invention, Montréal, P. Galluzzi, 1987

PIAGET Jean, Pour une Théorie de la Connaissance, Paris, Denoël Gonthier, 1970

RETI Ladislao, Léonard de Vinci, l'Artiste, l'Inventeur, Humaniste, Paris, Robert Laffont, 1974

RAVAISSON-MOLLIEN Charles, Les manuscrits de Léonard de Vinci, 6 tomes, Paris, A. Quantin, 1882-1891

ROBINSON Judith, L'analyse de l'esprit dans les Cahiers de Valéry, Paris, José Corti, 1963

SÉAILLES Gabriel, Léonard de Vinci, l'artiste et le savant. Essai de biographie psychologique, Paris, Perrin, 1892

SIMON Herbert Alexander, "L'unité des arts et des sciences : la psychologie de la pensée et de la découverte », texte présenté à l'Académie des sciences et des arts des Etats-Unis, 1959

SIMON Herbert Alexander, « Problems of Methodology Discussion », American Economic Review, 56, $\mathrm{n}^{\circ} 1,1963, \mathrm{p} .229-231$

SIMON Herbert Alexander, Les sciences de l'artificiel, Paris, Gallimard, (1969) 2004

VALÉRY Paul, «Introduction à la Méthode de Léonard de Vinci », La Nouvelle revue, t.95, 1895, p.

742-770 ; URL : http://catalogue.bnf.fr/ark:/12148/cb34356973m

VALÉRY Paul, « La conquête de l'ubiquité », Cuvres, Paris, Gallimard, Tome II, 1928

VALÉRY Paul, Vues, Paris, Éditions de la Table ronde, 1948

\section{NOTES}

1. http://www.intelligence-complexite.org/fileadmin/docs/2011hacomplexite.pdf

2. http://www.intelligence-complexite.org/nc/fr/documents/recherche-dun-document/doc/n1lunite-des-arts-et-des-sciences-la-psychologie-de-la-pensee-et-de-la-decouverte.html? tx_mcxapc_pi1\%5Baction\%5D=docDetail\&cHash=4e46081a280996e276a6739db7aa59ad

3. L'article de Paul Valéry a été répertorié par la Nouvelle revue, dans la table des matières tu tome 95, sous le titre: «L'esthétique de Léonard de Vinci» (https://gallica.bnf.fr/ark:/12148/ bpt6k360032/f877.item), note de la rédaction.

\section{RÉSUMÉS}

Léonard ne disposait pas des mots Technique et Technologique, mais il disposait d'un concept très riche, le mot Disegno qui en italien exprime à la fois le Dessein et le Dessin engagés dans l'invention de la Trans-Formation de Connaissances activantes. Réflexion qui nous reconduira à La Question de Paul Valéry: "Comment mon Esprit fonctionne?», laquelle nous incitera sans doute à associer aux recherches dites technologiques les recherches dans le champ des sciences de la cognition. Émergeront peut-être alors dans nos cultures générales les sciences d'Ingenium (ou en Sciences du Génie, G Vico, 1710) et les sciences de Conception (ou sciences de l'Artificiel, H. A. Simon 1969). 
Leonardo did not have the words Technical and Technological, but he had the very rich concept of Disegno, which expresses in Italian both Purpose and Drawing engaged in the invention of the Trans-activating Knowledge Training. A reflection that will lead us back to Paul Valery's Question "How does my mind work?", which will undoubtedly encourage us to combine so-called technological research with research in the field of the sciences of cognition. In our general cultures, the sciences of Ingenium - (or Engineering Sciences, G. Vico, 1710) and the Sciences of Design (or Artificial sciences, H. A. Simon, 1969) may then emerge.

\section{INDEX}

Mots-clés : histoire des techniques, histoire des sciences, cognition, conception, épistémologie, design, savoir, fonctionnalisme, ingénieur

Keywords : history of technology, history of science, epistemology, functionalism, cognition, design, knowledge, engineer

\section{AUTEUR}

\section{JEAN-LOUIS LE MOIGNE}

Professeur émérite de l'université d'Aix Marseille, ingénieur ECP, Jean-Louis Le Moigne est président de l'équipe d'animation du Réseau : http://www.intelligence-complexite.org/. Il a publié, entre autres, La théorie du système général : théorie de la modélisation en 1994 ; L'intelligence de la complexité. Épistémologie et pragmatique, de concert avec Edgar Morin, en 1999 ; Le Constructivisme, Tome 1. Les enracinements, Tome 2. Epistémologie de l'Interdisciplinarité, en 2001-2002. 